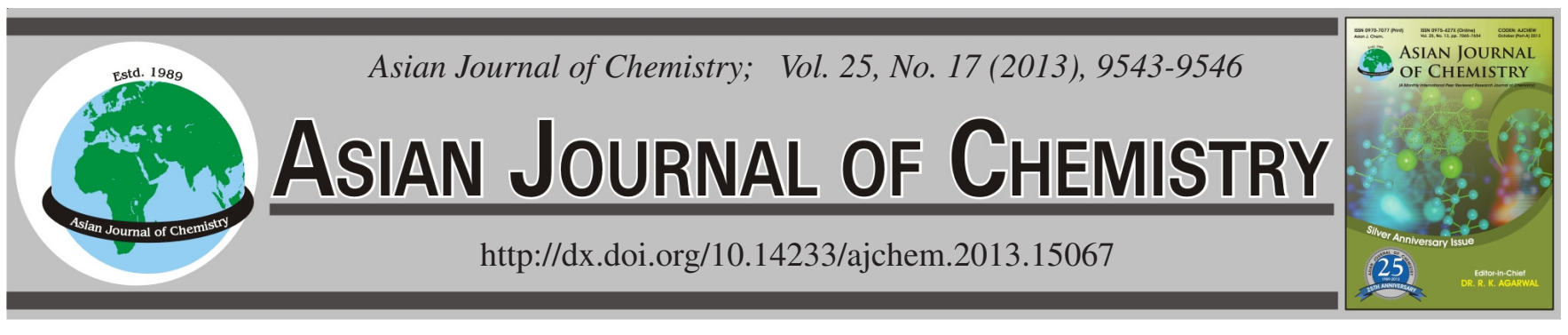

\title{
Treatment of Chemically Synthesized Polyaniline Wastewater by Combining Adsorption of Activated Carbon and Neutralization of Calcium Carbonate
}

\author{
QINGQING JU ${ }^{1,2}$, HAN HUANG ${ }^{1}$ and JiNQING KAN ${ }^{1, *}$
}

${ }^{1}$ School of Chemistry and Chemical Engineering, Yangzhou University, Yangzhou 225002, P.R. China

${ }^{2}$ Venturepharm Laboratories Limited, Taizhou City, 225316, P.R. China

*Corresponding author: E-mail: jqkan@yzu.edu.cn

(Received: 31 December 2012;

Accepted: 1 October 2013)

AJC-14224

\begin{abstract}
The purpose of this work was to study the treatment of polyaniline wastewater by adsorption of activated carbon and neutralization of calcium carbonate. The polyaniline wastewater generated in the synthesis process contained toxic aniline and its derivatives, which would pollute water and pose severe harm for human health and environment. Activated carbon is a strong adsorbent that could adsorb aniline and its derivatives effectively. Batch experiments such as the concentration of the polyaniline wastewater, adsorbent dose, contact time and $\mathrm{pH}$ were carried out to investigate the optimal treatment conditions. Results showed that the optimum conditions were at dilution concentration of 0.08 times of the raw concentration, $16.0 \mathrm{~g} \mathrm{~L}^{-1}$ activated carbon, $0.5 \mathrm{~h}$ and the original $\mathrm{pH} 1.05$. About $91 \%$ organics removal percentage could be reached by this method. Chemical oxygen demand (COD) of the polyaniline wastewater before and after treatment is 838 and $75 \mathrm{mg} \mathrm{L}^{-1}$, respectively and the removal efficiency was more than $91 \%$. The polyaniline wastewater after adsorption treatment was neutralized to $\mathrm{pH} 6.7$ by $8 \mathrm{~g} \mathrm{~L}^{-1} \mathrm{CaCO}_{3}$.
\end{abstract}

Key Words: Polyaniline wastewater, Activated carbon, Adsorption, Removal, Neutralization.

\section{INTRODUCTION}

Polyaniline (PANI) is a very promising polymer owing to its comparatively low cost, easy synthesis, good performance for temperature and reversible oxidation and reduction as well as good environmental stability ${ }^{1,2}$. Therefore, it has been extensively used in many fields such as rechargeable battery, sensor, electrode materials, electro-catalysis and anticorro$\operatorname{sion}^{3,4}$. Currently, there are mainly two kinds of polymerization of aniline, one is the chemical oxidation and the other is the electrochemical method ${ }^{5}$. The chemical oxidative polymerization of aniline using the ammonium persulfate as an oxidant in $\mathrm{H}_{2} \mathrm{SO}_{4}$ (or $\mathrm{HCl}$ ) aqueous solution is a suitable method for the mass production of polyaniline. The polymerization process is presented in Fig. 1.

But so far the wastewater produced during the synthesis process of PANI is less studied. The PANI wastewater containing toxic aniline and aniline derivatives (benzoquinone, hydrazobenzene, benzidine and ADPA), acids and inorganic salts can be highly toxic to human bodies ${ }^{7-9}$. It mainly enters the body through the skin, respiratory and digestive tract to undermine the blood resulting in hemolytic anemia and even leading to a variety of cancers. In addition, aniline and its derivatives also have a high acute toxicity to aquatic life and birds and the chronic toxic effects include shortened lifespan

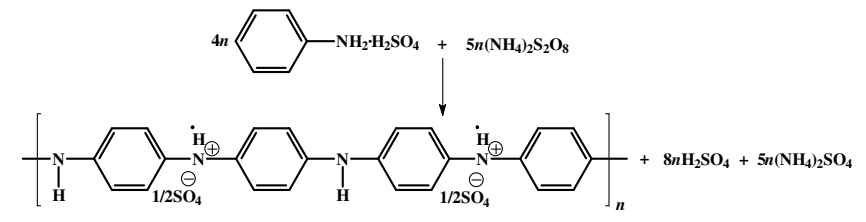

Fig. 1. Polymerization process of aniline [Ref. 6]

and changes in their appearance or behaviour ${ }^{10}$. Therefore, it is necessary and urgent to find an effective way to remove the undesirable organics before discharging to the environment.

An ideal wastewater treatment process must completely remove the toxic species without leaving behind hazardous residues and it should also be cost-effective. It is reported that there are mainly two ways to deal with the PANI wastewater. One is the cyclic utilization in which the filtrate could be used for many times till the acidic solution is completely consumed ${ }^{11}$. There are obvious problems in this method such as the acid concentration and oxidant dosage are difficult to be controlled. The other is by only using the adsorption of activated coal and adjusting $\mathrm{pH}$ to neutral with a relatively expensive alkaline $\left(\mathrm{Na}_{2} \mathrm{CO}_{3}\right)$ which will bring the impurities of sodium ions in the treated wastewater ${ }^{9}$. To the best of our knowledge, there have been no reports of the appropriate adsorption conditions for the PANI wastewater like concentration of PANI wastewater, adsorbent dose, contact time and $\mathrm{pH}$. 
Activated carbon is a preferred adsorbent for the removal of kinds of inorganic and organics from the aqueous solution ${ }^{12-14}$. In addition to the large adsorption area, activated carbon is almost nontoxic to the environment, relatively low cost and easily separated and recycled. It is reported that activated carbon has been widely used to adsorb aniline, phenol or their compounds wastewater ${ }^{15,16}$. However, the treatment of PANI wastewater by adsorption of activated carbon is less studied. Therefore, it is interesting and necessary to study the adsorption of PANI wastewater by activated carbon.

The acidic PANI wastewater must be adjusted to neutral before discharging to the environment. Calcium carbonate is a strong base-weak acid salt, which can neutralize the acidic wastewater. It is low cost than $\mathrm{Na}_{2} \mathrm{CO}_{3}$ or $\mathrm{NaOH}$ and the $\mathrm{SO}_{4}{ }^{2-}$ produced during the polymerization process could also be removed by reaction with $\mathrm{Ca}^{2+}\left(\mathrm{Ca}^{2+}+\mathrm{SO}_{4}{ }^{2-} \rightarrow \mathrm{CaSO}_{4} \downarrow\right)$. The calcium sulfate precipitates can be recycled as the raw material of chalk, paint, coatings, etc. The massive $\mathrm{CaCO}_{3}$ could be easily removed from the treated wastewater. Thus, it will be a preferred reagent for the neutralization of the acidic PANI wastewater.

In this paper, a cost-effective and simple method for the treatment of the PANI wastewater was studied by using the adsorption of activated carbon. We investigated the effects of concentration of the PANI wastewater, adsorbent dose, contact time and $\mathrm{pH}$ on the organics removal percentage of the PANI wastewater. The organics removal efficiency was further determined by chemical oxygen demand (COD). And the appropriate amount of massive $\mathrm{CaCO}_{3}$ for the neutralization of the acidic PANI wastewater was studied as well.

\section{EXPERIMENTAL}

Activated carbon powder was of analytical grade obtained from Kermel Reagent Co., Ltd. (Tianjin, China). All other chemicals such as aniline and ammonium persulfate were of analytical grade from Sinopharm Chemical Reagent Co., Ltd. (Shanghai, China). The aniline was distilled under reduced pressure prior to use. All solutions were prepared by using double-stilled water.

Wastewater preparation: To prepare PANI wastewater, $0.20 \mathrm{M}$ aniline and equal molar amount of ammonium persulfate was added into $0.50 \mathrm{M} \mathrm{H}_{2} \mathrm{SO}_{4}$ solutions with stirring. After reacting for $4 \mathrm{~h}$ at room temperature, the mixture was filtered and the filtrate namely the raw PANI wastewater was collected for further study.

Treatment of the PANI wastewater: As the absorbance of the raw PANI wastewater exceeded the measurement range of the UV spectrometer (Shimadzu, UV-2550), it was necessary to dilute the PANI wastewater before use. Assuming the concentration of the raw PANI wastewater was 1 , the dilution factor was $1 / 10$ to $1 / 250$ ( 0.004 to 0.1 times) of the raw solution $(25 \mathrm{~mL})$. Then we studied the absorbance change of the diluted solution by using UV spectrometer from 200-400 nm. After getting the appropriate dilution concentration, a series of equivalent diluted wastewater $(20 \mathrm{~mL})$ were first prepared and then different amounts of activated carbon were added to each solution to get the optimum adsorbent concentration. After adsorbing for $0.5 \mathrm{~h}$, the mixture was filtered and the absorbance of the filtrate was measured subsequently. The effects of contact time and $\mathrm{pH}$ on the fixed adsorbent concentration were investigated by using similar processes. The $\mathrm{pH}$ was adjusted to the desired level with the sodium hydroxide or sulfuric acid and measured by a PHS-25B pH meter (Shanghai Kanghua Biochemistry Instrument Co., Ltd., China). Blank samples were also run under same conditions. Each experiment was repeated and the results were taken as averages. The organics removal percentage was calculated by the following equation:

$$
\operatorname{Removal}(\%)=\frac{\left(\mathrm{A}_{0}-\mathrm{A}_{\mathrm{E}}\right)}{\mathrm{A}_{0}} \times 100 \%
$$

where removal (\%) is the organics removal percentage, $\mathrm{A}_{0}$ and $\mathrm{A}_{\mathrm{E}}$ are, respectively the initial and treated absorbance of the measured PANI wastewater. The organics removal efficiency was further confirmed based on the results of COD (Dichromate method, GB11914-89). The colour contrast images of the PANI wastewater before and after the treatment were taken by a Nokia C5-03. And the PANI wastewater after treatment was adjusted to neutral by a certain amount of massive $\mathrm{CaCO}_{3}$.

\section{RESULTS AND DISCUSSION}

UV absorption of polyaniline wastewater: The relationship between the concentration of the PANI wastewater and absorbance namely the calibration curve was shown in Fig. 2. The inset of Fig. 2 illustrated the UV spectra of the PANI wastewater at different dilution factor from $1 / 250$ to $1 / 10(0.004$ to 0.1 times) of the raw PANI wastewater. It could be seen from the inset of Fig. 2 that the PANI wastewater had a strong, steady and characteristic absorption peak at $287 \mathrm{~nm}$, which was consistent with the literature ${ }^{9,17}$. It was noticed that the dilution concentration of the raw PANI wastewater was in linear correlation with the absorbance $\left(y=7.4468 x+0.0319, R^{2}=\right.$ 0.9971), which obeyed the Lambert-Beer's law in a certain concentration range. So the concentration of the organics in the PANI wastewater could be determined by UV spectrometer. Besides, it could be concluded that 2/25 (0.08 times) of the raw concentration was an appropriate concentration for the following study where the initial absorbance was 0.666 .

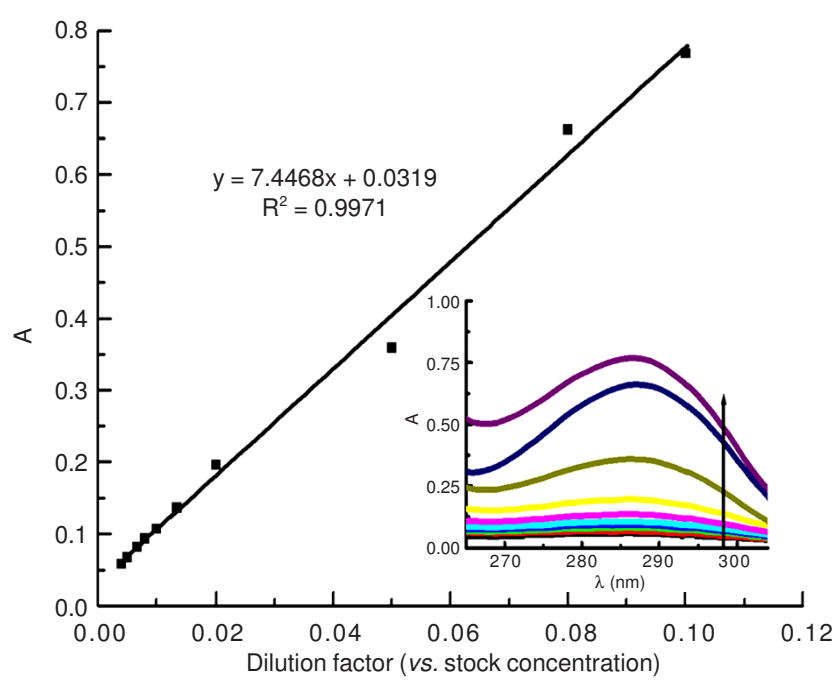

Fig. 2. Calibration curve of the PANI wastewater 
Effect of adsorbent dose: The adsorbent dosage was an important parameter for adsorption reaction because a given amount of adsorbent could only adsorb a fixed amount of adsorbate from the solution. Different amount of adsorbent from 5.0-27.5 $\mathrm{g} \mathrm{L}^{-1}$ was added into the $20 \mathrm{~mL}$ diluted PANI wastewater $(0.08$ times of the raw PANI wastewater concentration). After adsorbing for $0.5 \mathrm{~h}$ at room temperature, the absorbance of the treated wastewater was detected by the UV spectrometer and the removal percentage was calculated by above equation $\left(\right.$ removal $\left.(\%)=\left(\mathrm{A}_{0}-\mathrm{A}_{\mathrm{E}}\right) / \mathrm{A}_{0} \times 100 \%\right)$. The effect of adsorbent dose on removal percentage was shown in Fig. 3. It was noticed that the organics removal percentage of the PANI wastewater increased from 61 to $92 \%$ with increasing adsorbent dose from 5.0 to $27.5 \mathrm{~g} \mathrm{~L}^{-1}$, respectively. The high removal percentage was because more adsorption sites were available for the interaction of adsorbent with the organics in the PANI wastewater. However, when the adsorbent dose increased over $16 \mathrm{~g} \mathrm{~L}^{-1}$, the removal percentage showed no significant change. It might be due to the overlapping of the active sites at higher dosage resulting in lowering of the net surface area of the adsorbent ${ }^{18}$. So, $16 \mathrm{~g} \mathrm{~L}^{-1}$ was supposed as the optimum concentration of activated carbon.

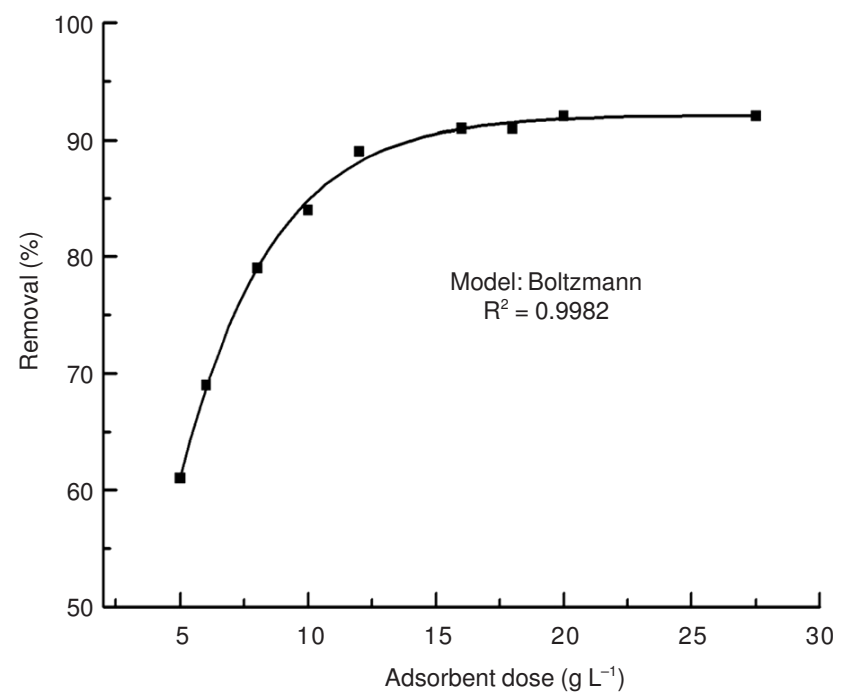

Fig. 3. Effect of adsorbent dose on organics removal percentage $(20 \mathrm{~mL}$ wastewater, $0.5 \mathrm{~h}$, room temperature)

Effect of contact time: The effect of contact time on the removal percentage of the organics of the PANI wastewater was further investigated. The result was shown in Fig. 4. The removal percentage of the organics increased from 79 to $92 \%$ with increasing contact time from 5 to $60 \mathrm{~min}$. The main adsorption of the organics in the PANI wastewater occurred at the beginning $0.5 \mathrm{~h}$. Within $0.5 \mathrm{~h}$, the removal percentage of organics reached $91 \%$, which showed relatively high organics removal efficiency. Initially, the activated carbon adsorption processes of organics were rapid because the surfaces of activated carbon were not occupied by any organics in the PANI wastewater so as the organics struck the surfaces of activated carbon might get adsorbed immediately ${ }^{19}$. Afterwards, the adsorption processes became slow due to that the adsorption of organics by activated carbon reached saturation gradually. Thus the best adsorption time period $(0.5 \mathrm{~h})$ was suggested.

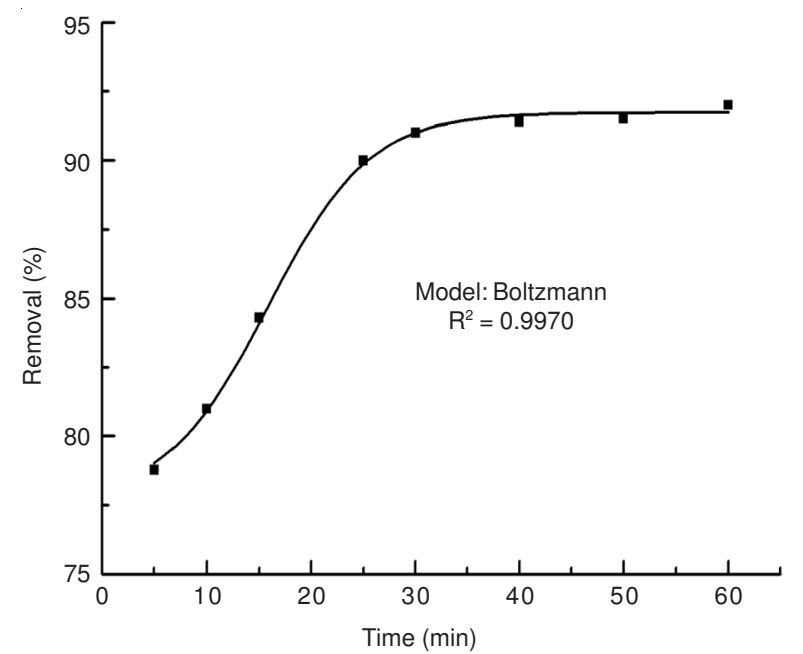

Fig. 4. Effect of contact time on organics removal percentage $(20 \mathrm{~mL}$ wastewater, $16 \mathrm{~g} \mathrm{~L}^{-1}$ activated carbon, room temperature)

Effect of pH: To investigate the effect of $\mathrm{pH}$ on the organics removal percentage of the PANI wastewater, the wastewater was first adjusted to the $\mathrm{pH}$ range from 0.95 to 9 and then treated by keeping all other parameters constant. The organics removal percentage at $\mathrm{pH}$ from 0.95 to 9 was illustrated in Fig. 5 where the $\mathrm{pH}$ of the diluted PANI wastewater was 1.05. The removal percentage showed no apparent change from $\mathrm{pH} 0.95$ to 2 and then decreased when $\mathrm{pH}$ increased over 2 . Among it, the organics removal percentage reached its maximum of $c a .91 \%$ at $\mathrm{pH}$ 0.95-2.00. As we further increased the solution $\mathrm{pH}$, removal percentage of the PANI wastewater decreased. The organics removal percentage in the PANI wastewater decreased under neutral and basic conditions, which might be due to the competition between the $\mathrm{OH}^{-}$ions and the adsorbents molecules for the adsorption sites of activated carbon $^{20}$. The results demonstrated that activated carbon had a higher amount of adsorption under acidic than under basic conditions, which was consistent with the literature ${ }^{21}$. Therefore, the original $\mathrm{pH} 1.05$ was an appropriate $\mathrm{pH}$ condition for the treatment of the PANI wastewater.

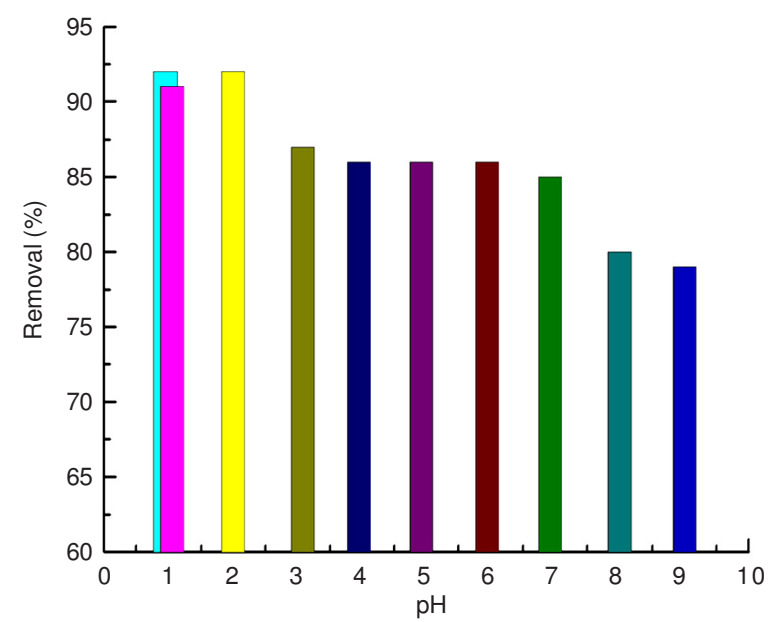

Fig. 5. Effect of $\mathrm{pH}$ on organics removal percentage $(20 \mathrm{~mL}$ wastewater, $16 \mathrm{~g} \mathrm{~L}^{-1}$ activated carbon, $0.5 \mathrm{~h}$ and room temperature)

Measurement of COD: COD is a typical way to determine the content of organic materials in the wastewater. The COD 
of the adsorption treatment PANI wastewater was $75 \mathrm{mg} \mathrm{L}^{-1}$, which met level 2 of Discharge Standard of Pollutants for Municipal Wastewater Treatment Plant (GB 18918-2002). As the COD of the PANI wastewater was $838 \mathrm{mg} \mathrm{L}^{-1}$, the COD removal efficiency was more than $91 \%$, which further confirmed that the organics in the PANI wastewater was nearly completely removed by the adsorption of activated carbon.

Colour contrast images of the PANI wastewater before and after treatment: Image (a) and (b) in Fig. 6 represented the wastewater before treatment and wastewater after treatment, respectively. The colour of solutions changed obviously from original deep purple of image (a) to final colourless of image (b). The deep colour of image (a) might be due to the large amount of dissolved organics in the PANI wastewater before treatment. As activated carbon could adsorb the residual organics effectively, the final solution turned from deep purple to final colourless.

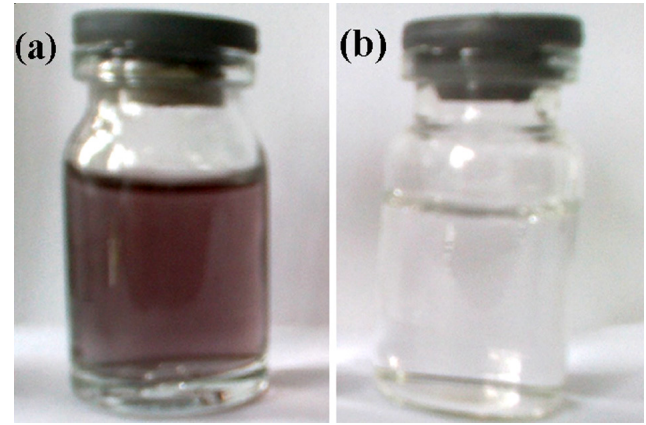

Fig. 6. Colour contrast images of the PANI wastewater before and after treatment (a) wastewater before treatment (b) wastewater after treatment

pH adjustment of the adsorption treatment PANI wastewater: The colourless PANI wastewater after adsorption treatment was further adjusted to neutral by using a series of different amount of massive $\mathrm{CaCO}_{3}$. The relationship between $\mathrm{CaCO}_{3}$ dosage and the final solution $\mathrm{pH}$ was shown in Fig. 7. It could be seen that the optimum $\mathrm{CaCO}_{3}$ dosage for the neutralization of the PANI wastewater was $8 \mathrm{~g} \mathrm{~L}^{-1}$ where the $\mathrm{pH}$ was 6.70 .

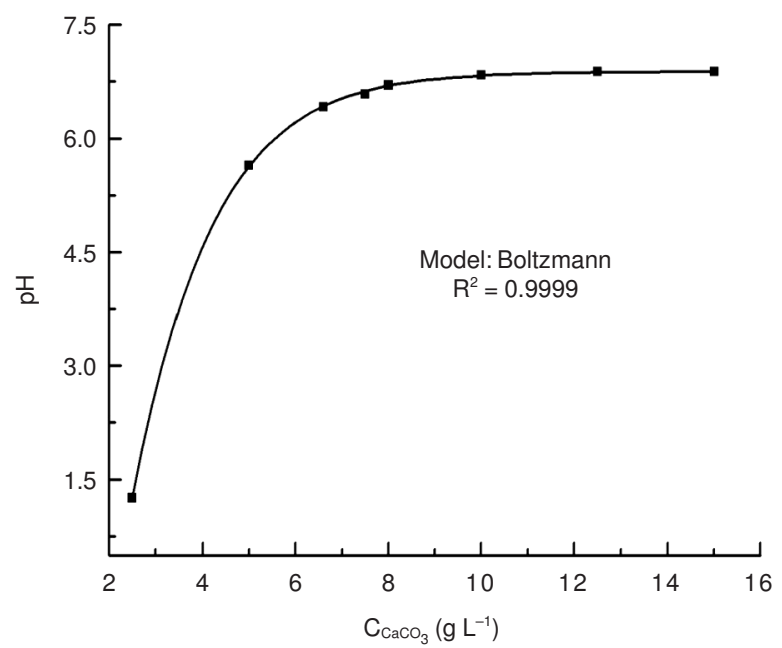

Fig. 7. Relationship between $\mathrm{CaCO}_{3}$ dosage and the final solution $\mathrm{pH}$

\section{Conclusion}

The results showed that the optimum adsorption conditions of the PANI wastewater were 0.08 times of the raw concentration, $16.0 \mathrm{~g} \mathrm{~L}^{-1}$ activated carbon, $0.5 \mathrm{~h}$ and the original $\mathrm{pH}$ 1.05. A relatively complete organics removal percentage of the PANI wastewater ( $c a .91 \%$ ) could be reached in this method. Chemical oxygen demand (COD) of the PANI wastewater before and after treatment is 838 and $75 \mathrm{mg} \mathrm{L}^{-1}$, respectively and the removal efficiency of the PANI wastewater was more than $91 \%$. The final PANI wastewater was neutralized to $\mathrm{pH} 6.70$ by $8 \mathrm{~g} \mathrm{~L}^{-1} \mathrm{CaCO}_{3}$. It is a new method to deal with the PANI wastewater with low-cost, simple process and significant effect. Therefore, this method may have broad application prospects in the near future.

\section{ACKNOWLEDGEMENTS}

This project was supported by National Science Foundation of China (No. 20873119) and by the Priority Academic Program Development of Jiangsu Higher Education Institutions. Part of data was from Testing Center of Yangzhou University.

\section{REFERENCES}

1. F. Karci and M. Kabasakaloglu, Asian J. Chem., 25, 2533 (2013).

2. S. Li, L. Tang, R. Tang and J. Kan, Asian J. Chem., 22, 415 (2010).

3. R. Ansari and A.H. Alikhani, J. Coat. Technol. Res., 6, 221 (2009).

4. I. Sapurina and J. Stejskal, Chem. Pap., 63, 579 (2009).

5. J.X. Huang and R.B. Kaner, Chem. Commun., 367 (2006).

6. R. Ansari and Z. Mosayebzadeh, Chem. Pap., 65, 1 (2011).

7. A. Mohammad, Inamuddin and A. Amin, J. Therm. Anal. Calorim., 107, 119 (2012).

8. Y. Sun, L.Y. Liang, X.Y. Zhao, L. Yu, J. Zhang, G.Y. Shi and T.H. Zhou, Water Res., 43, 41 (2009).

9. R.A. Sousa, O.A. Araújo, P.S. Freitas and M.A. De Paoli, Quim. Nova, 26, 938 (2003).

10. S. Hawkins and Y. Waguespack, J. Undergrad. Chem. Res., 2, 51 (2003).

11. K.S. Ho and T.H. Hsieh, U.S. Patent 5959072 (1999).

12. J.M. Dias, M.C. Alvim-Ferraz, M.F. Almeida, J. Rivera-Utrilla and M. Sánchez-Polo, J. Environ. Manage., 85, 833 (2007).

13. D.Z. Chen, J.X. Zhang and J.M. Chen, Int. J. Environ. Sci. Technol., 7, 235 (2010).

14. J.M. Salman, F.M. Abid and A.A. Muhammed, Asian J. Chem., 24, 5646 (2012).

15. K. László, Colloids Surf. A, 265, 32 (2005).

16. B. Tang, Y.W. Lin, P. Yu and Y.B. Luo, Chem. Eng. J., 187, 69 (2012).

17. J.Q. Kan and S.L. Mu, Acta Polym. Sin., 4, 468 (1989).

18. S. Kagne, S. Jagtap, D. Thakare, S. Devotta and S.S. Rayalu, Desalination, 23, 26 (2009).

19. G.Q. Wu, X. Zhang, H. Hui, J. Yan, Q.S. Zhang, J.L. Wan and Y. Dai, Chem. Eng. J., 185, 206 (2012).

20. B. Tang, Y.W. Lin, P. Yu and Y.B. Luo, Chem. Eng. J., 187, 71 (2012).

21. L.X. Zheng, S.L. Su, X. Y. Chang, L.X. Yang, X.T. Liu and L.N. Jin, Environ. Sustain. Dev., 20, 2 (2010). 